\title{
A ROBUST MODEL-BASED ALGORITHM FOR LOCALIZING MARINE MAMMAL TRANSIENTS
}

\author{
CHRISTOPHER O. TIEMANN, MICHAEL B. PORTER \\ Science Applications International Corporation \\ 1299 Prospect St, Suite 303, La Jolla, CA 92037, USA \\ JOHN A. HILDEBRAND \\ Scripps Institution of Oceanography \\ University of California at San Diego, La Jolla, CA 92093, USA
}

\begin{abstract}
Given the increasing interest in the effects of sound on marine mammals, a new modelbased algorithm for localizing transient noises around a sparse, widely-distributed array of receivers has been developed and tested with real acoustic data. The robustness of the algorithm is illustrated in its application to two different scenarios with equal success: localizing humpback whales near Hawaii and blue whales near California. The algorithm is novel in its use of acoustic propagation modeling and in construction of an ambiguity surface to identify the most probable whale location in a horizontal plane around an array. A description of the algorithm and examples of its application to marine mammal localization are provided.
\end{abstract}

\section{Introduction}

In 1996, twelve beaked whales stranded themselves in Greek coastal waters while a low-frequency active SONAR system was being tested by NATO. In March 2000, a similar event happened in the Bahamas during a U.S. naval exercise [1]. While bi-catch (incidental capture by fishing vessels) is probably the major threat to marine mammals, these stranding incidents have generated an increased sensitivity to the possible effects of sound on the marine environment.

Three directions are being pursued to mitigate these problems: 1) study the behavior of marine mammals, 2) detect the presence of marine mammals in an area where SONAR is being operated, and 3) adjust power output or operating areas of SONAR systems. Our research here focuses principally on the first two areas and especially on navy ranges. Interestingly, the calls of many of these animals are often easily heard underwater. Indeed some marine mammals call at levels easily exceeding operational SONAR (for instance, the bottlenose dolphin is estimated to produce clicks at about $220 \mathrm{~dB}$ ) [2]. This suggests the possibility of using passive acoustic techniques to both detect the presence of marine mammals and observe their behavior. From a signal processing point of view, the problem is one of detecting and localizing transients on an extremely sparse (i.e. widely distributed) set of hydrophones, but such a technique must be robust against variability typical of the littoral environment in which many marine mammals exist. 


\section{TIEMANN ET AL.}

We developed an algorithm for this purpose and initially applied it to tracking humpback whales near the Pacific Missile Range Facility (PMRF) hydrophone array in waters near Kauai, Hawaii [3]. More recently, we have had the opportunity to test the algorithm using data from ocean bottom seismometers (OBS) deployed in shallow waters off the coast of southern California where the key species of interest is blue whales. The calls of blue whales are very loud, low frequency rumbles with a strong $10-20 \mathrm{~Hz}$ component and duration of about $20 \mathrm{sec}$. This is quite different from the brief $(1-2 \mathrm{sec})$, high-frequency $(.2-4 \mathrm{kHz})$ calls of the humpbacks. Nevertheless, the same algorithm provides reliable tracking of both species despite the markedly different call characteristics and environments under study. In the remaining sections, we present the localization method and describe its application to both sites.

\section{Localization Algorithm}

In past studies of marine mammal behavior, the typical method of localizing animals through passive acoustic means used hyperbolic fixing [4-9]. This technique uses the measured difference in arrival time (or time-lag) of a whale call recorded on multiple hydrophone pairs to produce intersecting hyperbolas indicating the animal's position. However, the assumption of a constant soundspeed inherent to hyperbolic fixing can sometimes prevent an accurate localization, particularly at longer ranges [3].

The algorithm we present here has several novel features and advantages over other localization methods. It is based on an acoustic propagation model to account for variations in soundspeed and bathymetry, thus eliminating the constant soundspeed errors associated with hyperbolic fixing. Its output is a graphical display that easily conveys the source location and confidence in the estimate. It adds robustness against environmental variability and acoustic multipath by performing some processing in the spectral domain $[6,8]$. Lastly, the algorithm is also suitable for continuous, real-time operation without user interaction.

The localization algorithm consists of two main components: spectral pattern correlation to calculate pair-wise time-lags, plus an ambiguity surface construction to generate a location estimate.

\subsection{Spectrogram Correlation}

Measuring time-lags between whale call arrivals for all hydrophone pair combinations is a critical step in the algorithm. Typically this is done through cross correlation, but whether the correlation should be performed on the original waveforms or their spectrograms is open to debate $[6,8]$. In analysis of data from PMRF, best results were obtained by following an example of spectral shape correlation described by Seem and Rowe [10]. Spectrograms from two hydrophones are digitized, i.e. converted to two levels of intensity (on or off) based on a data-adaptive threshold. As two digitized spectrograms are shifted past each other, correlation is performed very quickly by a logical AND of the overlapping regions. Summing the overlapping pixels provides a correlation score whose maximum determines the time-lag between channels.

To demonstrate the strength of the spectral correlation method, an example of cross correlator output using both waveform and spectral correlation is shown in Fig. 1. The data are from two hydrophones of the PMRF data set for minute 20:16 on March 22, 
2001. A time window 10 seconds long extracts data subsets to use with each correlation; the window advances in 1-second increments, calculating a time-lag at each step. The waveform correlation's time-lag estimates are quite variable over the minute, perhaps due to interferers such as other distant animals singing simultaneous songs [6]. Nevertheless, the spectral correlation process correctly extracts the pair-wise time-lag of 7 seconds during periods when a whale is clearly singing.
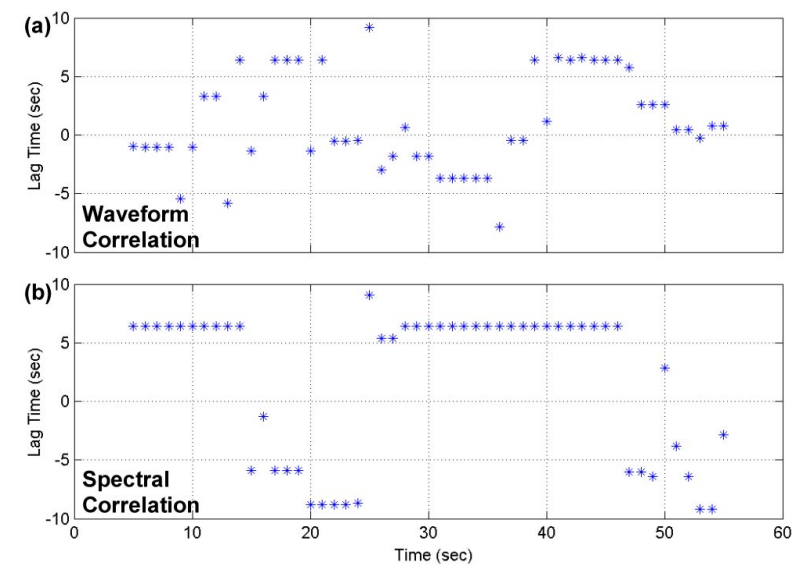

Figure 1. Time-lag measurements from waveform (a) and digitized spectrogram (b) cross correlation. Data is from PMRF hydrophones 2 and 5 for minute 20:16 on 3/22/01. The consistency of the spectral correlator made it the preferred method for time-lag measurements.

\subsection{Ambiguity Surface Construction}

A singing whale is localized through the construction of an ambiguity surface, which is a probabilistic indicator of source position made through the comparison of measured time-lags ('data') to predicted time-lags ('replicas'). The first step in creation of the replica is to calculate direct path acoustic travel times from a grid of possible source positions within a $30 \mathrm{~km}$ square area to every hydrophone. The acoustic propagation model BELLHOP [11] was used to calculate travel times as it can account for depthdependent soundspeed profiles and varying receiver depths. Historic average soundspeed profiles were used in the acoustic modeling. When localizing humpback whales, a $500-\mathrm{Hz}$ source at $10-\mathrm{m}$ depth was assumed during replica generation; for blue whales a $30-\mathrm{Hz}$ source at $35-\mathrm{m}$ depth was assumed. The replica is computed by calculating the difference in travel times from a hypothesized source position to a pair of receivers.

For each candidate source position, the predicted time-lag that would be seen by a receiver pair is then compared to the measured time-lag for that pair to determine the likelihood that the source is at that position. A likelihood score is then scaled according to the acoustic transmission loss predicted by BELLHOP, thus minimizing the likelihood of a detection at long range from the array. Likelihood scores are then assembled on a two-dimensional horizontal plane around the array, completing one ambiguity surface. Ambiguity surfaces based on time-lag estimates with high 


\section{TIEMANN ET AL.}

correlation scores are summed to make an overall ambiguity surface where source location estimates common to many receiver pairs stack to form a peak. The ambiguity surface peak is declared the best estimate of source position.

\section{Localizations at PMRF}

The Pacific Missile Range Facility has an underwater array of over 100 bottom mounted hydrophones, and a system has been implemented for transferring acoustic data from 6 hydrophones to the Maui High-Performance Computing Center (MHPCC) for analysis. The hydrophones are spaced 5-20 km apart and are shown on the contour map of Fig. 2 . Two days of acoustic data from March 2001 were used for algorithm development. Humpback whale songs were heard on every hydrophone channel at all times of day; sometimes multiple marine mammals could be heard on the same channel simultaneously.

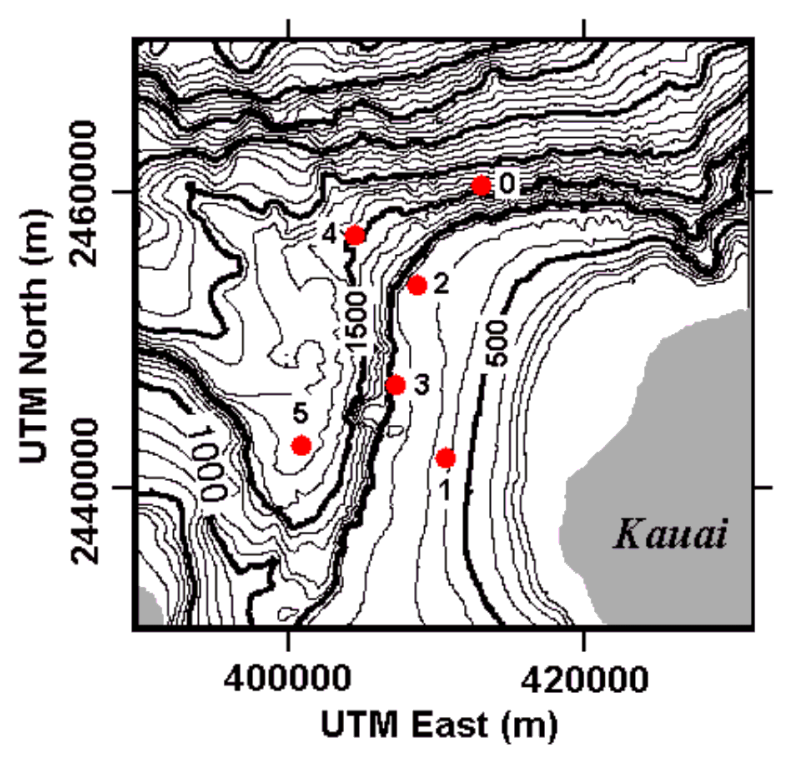

Figure 2. Bathymetry contours $(\mathrm{m})$ and hydrophone locations $(0-5)$ at the Pacific Missile Range Facility. Axes are for UTM Zone 4.

An example of a humpback whale localization through ambiguity surface construction is shown in Fig. 3. The ambiguity surface represents a $30-\mathrm{km}$ square area around the PMRF array with hydrophone positions labeled. Bright areas on the surface represent likely whale positions, with the peak indicated by a crosshair designating the animal's location. The ambiguity surfaces reveal patterns that resemble hyperbolas, but they have been thickened and stacked so that the most probable source location can be automatically extracted. This analysis was applied to many time segments throughout the data set, and in every case, a source was localized by the contribution of four or more receiver pairs. 


\section{LOCALIZING MARINE MAMMAL TRANSIENTS}



Figure 3. Ambiguity surface showing a plan view of the waters around the PMRF array with hydrophone positions (0-5) indicated. Axes are for UTM Zone 4. Intensity peak and crosshair indicate whale position estimate. Data is from 3/22/01 20:16.

\section{Localizations at San Clemente}

Twelve hours of data from four seismometers west of San Clemente Island were used for further algorithm testing. The seismometers were deployed on the seafloor at about $200 \mathrm{~m}$ depth in a $3 \mathrm{~km}$ square, as shown in Fig. 4. The long, low frequency calls of blue whales were heard on every channel during most of the data set. Because of the much longer call duration of blue whales, the correlation window size was increased from 10 seconds to 60 seconds. Doing so allows the spectral correlator to see more distinguishing spectral patterns with each iteration. This was the only change made to the algorithm. After replicas were generated for the new array geometry, the localization process was run again.

An example ambiguity surface showing a single blue whale localization appears in Fig. 5. This surface represents a $20 \mathrm{~km}$ square plan view around the seismometer array. Again, the bright peak and crosshair indicate a likely whale location. During times when only one blue whale was heard singing, it was easy to follow its motion through the array with consecutive localizations. To illustrate this, Fig. 6 shows two track estimates from August 28, 2001, on $10 \mathrm{~km}$ square plan views around the seismometer array. In Fig. 6a, a whale is swimming across the array from north to south at approximately $7 \mathrm{~km} / \mathrm{hr}$. Figure $6 \mathrm{~b}$ tracks a whale reversing direction. 


\section{TIEMANN ET AL.}

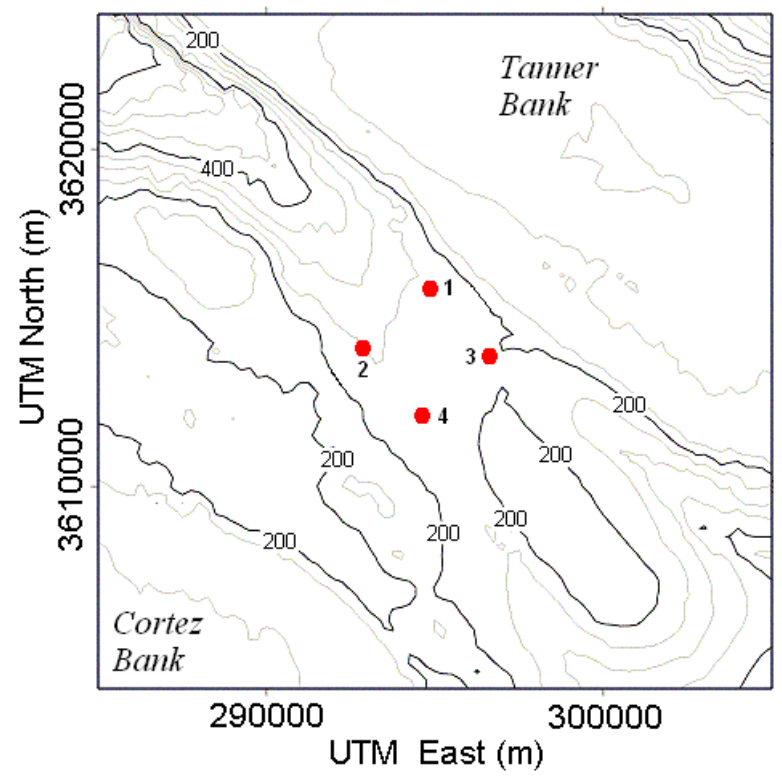

Figure 4. Bathymetry contours (m) and seismometer locations (1-4) near San Clemente Island off southern California. Axes are for UTM Zone 11.



Figure 5. Ambiguity surface showing a plan view of the waters around the San Clemente array with seismometer positions (1-4) indicated. Axes are for UTM Zone 11. Intensity peak and crosshair indicate whale position estimate. Data is from 8/28/01 05:36. 


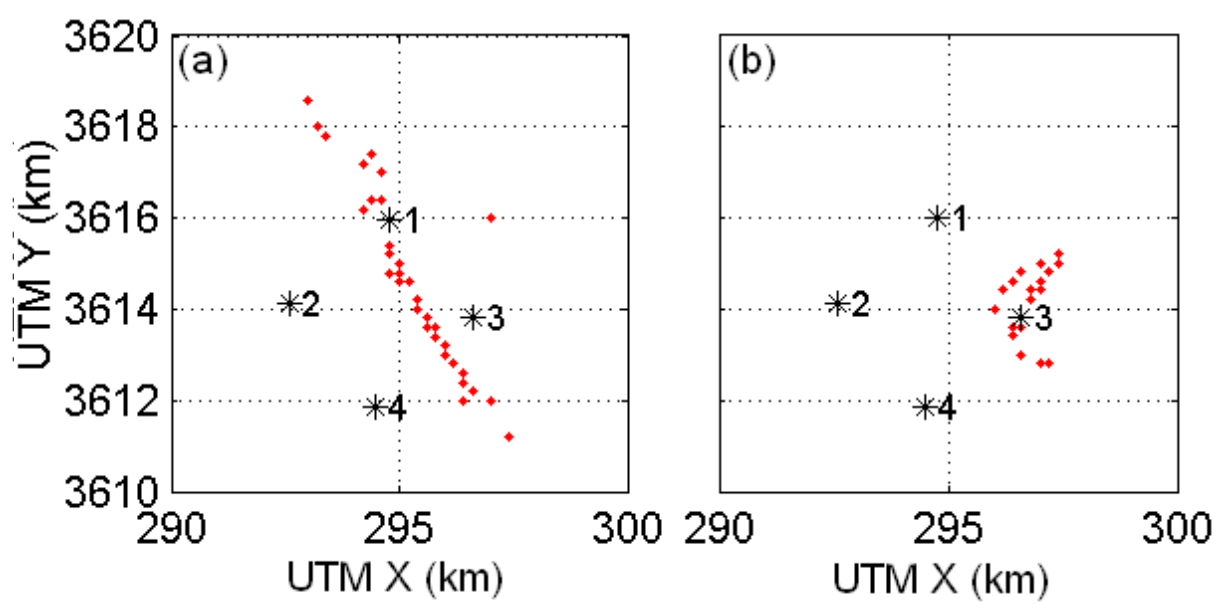

Figure 6. Plan view of the waters around the San Clemente array with seismometer positions (1-4) indicated. Axes are for UTM Zone 11. Points show blue whale location estimates from 8/28/01. (a) From 03:00 to 04:13 a whale moved from northwest to southeast over the array.

(b) From 10:10 to 12:00 a whale swam northeast over sensor 3, then reversed direction.

\section{Discussion}

The model-based localization technique described here could be a valuable tool for those wishing to study marine mammal behavior or minimize the effects of sound on such animals. When applied to long records, the algorithm has the potential to deliver useful information of whale behavior, such as in visualizing migration paths. However, its real benefit could be for those interested in mammal mitigation issues through continuous, real-time, automated monitoring applications. In tests with the PMRF data set, the computations could be completed within the data update period, and setting high thresholds on scoring would minimize chances of false alarms.

The algorithm's success in two different scenarios hints at its robustness against environment, target type, and array geometry. It is also readily portable to any array shape and may work equally well localizing other transient audible targets, both natural and manmade. Because localization is now limited to a single assumed depth, future enhancements could include taking better advantage of depth-dependent multipath structure to generate a depth estimate. Confirmation of the algorithm through nonacoustic means, such as by concurrent visual observations, is another goal. 


\section{TIEMANN ET AL.}

\section{Acknowledgements}

We gratefully acknowledge the help of the following people from PMRF, MHPCC, and SAIC in providing and preparing the Hawaii data for use: Jim Hagar, Robert Desonia, D.J. Fabozzi, and Paul Hursky. Thanks to Allan Sauter of SIO for preparing the San Clemente OBS data for use. Neil Frazer and Herb Freese contributed in many helpful discussions, and Richard Bachman provided environmental characterizations of both sites. This work was supported by the National Defense Center of Excellence for Research in Ocean Sciences (CEROS contract 47316). Additional support was provided by Office of Naval Research (ONR contract N00014-00-D-0115).

\section{References}

1. U.S. Department of Commerce and Department of the Navy, "Joint Interim Report: Bahamas Marine Mammal Stranding Event of 15-16 March 2000" (2001).

2. W. J. Richardson, C. R. Greene, Jr., C. I. Malme, and D. H. Thomson, Marine Mammals and Noise (Academic Press, 1995), p. 185.

3. C. O. Tiemann, M. B. Porter, and L. Neil Frazer, "Automated model-based localization of marine mammals near Hawaii," MTS/IEEE Oceans 2001 Conference Proceedings, Honolulu, Hawaii, November 5-8, 2001 (Holland Publications, 2001), pp: 1395-1400.

4. S. Mitchell and J. Bower, "Localization of animal calls via hyperbolic methods," J. Acoust. Soc. Am. 97, 3352-3353 (1995).

5. A. S. Frankel, C. W. Clark, L. M. Herman, and C. M. Gabriele, "Spatial distribution, habitat utilization, and social interactions of humpback whales, Megaptera novaeangliae, off Hawai'i determined using acoustic and visual techniques," Can. J. Zool. 73, 1134-1146 (1995).

6. V. M. Janik, S. M. Van Parijs, and P. M. Thompson, "A two-dimensional acoustic localization system for marine mammals," Mar. Mamm. Sci. 16, 437-447 (2000).

7. K. M. Stafford, C. G. Fox, and D. S. Clark, "Long-range acoustic detection and localization of blue whale calls in the northeast Pacific Ocean," J. Acoust. Soc. Am. 104(6), 36163625 (1998).

8. C. W. Clark and W. T. Ellison, "Calibration and comparison of acoustic location methods used during the spring migration of the bowhead whale, Balaena mysticetus, off Pt. Barrow, Alaska, 1984-1993,” J. Acoust. Soc. Am. 107(6), 3509-3517(2000).

9. C. W. Clark, W. T. Ellison, K. Beeman, "Acoustic tracking of migrating bowhead whales," IEEE Oceans 1986 Conference Proceedings, 341-346.

10. D. A. Seem and N. C. Rowe, "Shape correlation of low-frequency underwater sounds," $J$. Acoust. Soc. Am. 95(4), 2099-2103 (1994).

11. M. B. Porter and Y. C. Liu, "Finite-Element Ray Tracing", Proceedings of the International Conference on Theoretical and Computational Acoustics, Eds. D. Lee and M. H. Schultz (World Scientific, 1994), pp. 947-956. 